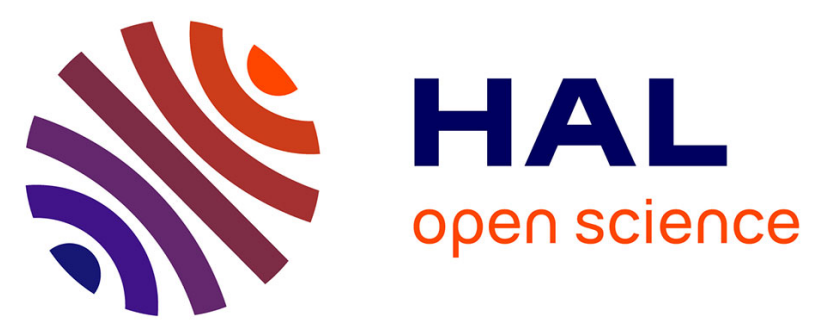

\title{
Genomic evolution of the globally disseminated multidrug-resistant Klebsiella pneumoniae clonal group 147
}

Carla Rodrigues, Siddhi Desai, Virginie Passet, Devarshi Gajjar, Sylvain Brisse

\section{To cite this version:}

Carla Rodrigues, Siddhi Desai, Virginie Passet, Devarshi Gajjar, Sylvain Brisse. Genomic evolution of the globally disseminated multidrug-resistant Klebsiella pneumoniae clonal group 147. Microbial Genomics, 2022, 8 (1), 10.1099/mgen.0.000737 . pasteur-03566824

\section{HAL Id: pasteur-03566824}

https://hal-pasteur.archives-ouvertes.fr/pasteur-03566824

Submitted on 11 Feb 2022

HAL is a multi-disciplinary open access archive for the deposit and dissemination of scientific research documents, whether they are published or not. The documents may come from teaching and research institutions in France or abroad, or from public or private research centers.
L'archive ouverte pluridisciplinaire HAL, est destinée au dépôt et à la diffusion de documents scientifiques de niveau recherche, publiés ou non, émanant des établissements d'enseignement et de recherche français ou étrangers, des laboratoires publics ou privés.

\section{(c)(1)}

Distributed under a Creative Commons Attribution| 4.0 International License 
5 Carla Rodrigues ${ }^{1}$, Siddhi Desai ${ }^{2}$, Virginie Passet ${ }^{1}$, Devarshi Gajjar ${ }^{2}$ and Sylvain Brisse ${ }^{1 *}$

6

$7 \quad{ }^{1}$ Institut Pasteur, Biodiversity and Epidemiology of Bacterial Pathogens, Paris, France.

$8 \quad{ }^{2}$ Department of Microbiology and Biotechnology Centre, Faculty of Science, The Maharaja

9 Sayajirao University of Baroda, Vadodara, Gujarat, India.

10

$11 *$ Corresponding author:

12 Sylvain Brisse

13 Institut Pasteur,

14 Biodiversity and Epidemiology of Bacterial Pathogens,

1528 rue du Docteur Roux, F-75724 Paris, France.

16 E-mail: sylvain.brisse@pasteur.fr;

17 Phone: +3314568 8334

18

19 Keywords: phylogenomics, pandrug resistance, last-resort antibiotics, high-risk clone,

20 NDM-5, CRISPR/Cas systems, IncF plasmids, genomic epidemiology

22 Repositories: Reads and genomic sequences of the isolates analysed in this study were

23 deposited at the European Nucleotide Archive (accession number PRJEB41234). 
ABSTRACT

The rapid emergence of multidrug-resistant Klebsiella pneumoniae (Kp) is largely driven by the spread of specific clonal groups (CG). Of these, CG147 includes 7-gene MLST sequence types ST147, ST273 and ST392. CG147 has caused nosocomial outbreaks across the world, but its global population dynamics remain unknown. Here, we report a pandrugresistant ST147 clinical isolate from India (strain DJ) and define the evolution and global emergence of CG147. Antimicrobial susceptibility testing (EUCAST guidelines) and genome sequencing (Illumina and Oxford Nanopore Technologies, Unicycler assembly) were performed on strain DJ. Additionally, we collated 217 publicly available CG147 genomes (NCBI, May 2019). CG147 evolution was inferred within a temporal phylogenetic framework (BEAST) based on a recombination-free sequence alignment (Roary/Gubbins). Comparative genomic analyses focused on resistance and virulence genes and other genetic elements (BIGSdb, Kleborate, PlasmidFinder, PHASTER, ICEFinder and CRISPRCasFinder).

Strain DJ had a pandrug resistance phenotype. Its genome comprised 7 plasmids and 1 linear phage-plasmid. Four carbapenemase genes were detected: $b l a_{\mathrm{NDM}-5}$ and 2 copies of bla $a_{\mathrm{OXA}-181}$ in the chromosome, and a second copy of bla $a_{\mathrm{NDM}-5}$ on an $84 \mathrm{~kb}$ IncFII plasmid. CG147 genomes carried a mean of 13 acquired resistance genes or mutations; $63 \%$ carried a carbapenemase gene and 83\% harbored bla $a_{\mathrm{CTX}-\mathrm{M}}$. All CG147 genomes presented GyrA and ParC mutations and a common subtype IV-E CRISPR-Cas system. ST392 and ST273 emerged in 2005 and 1995, respectively. ST147, the most represented phylogenetic branch, was itself divided into two main clades with distinct capsular loci: KL64 (74\%, DJ included, emerged in 1994 and disseminated worldwide, with carbapenemases varying among world regions) and KL10 (20\%, 2002, predominantly found in Asian countries, associated with carbapenemases NDM and OXA-48-like). Further, subclades within ST147- 
49 KL64 differed at the yersiniabactin locus, OmpK35/K36 mutations, plasmid replicons and

50 prophages. The absence of IncF plasmids in some subclades was associated with a possible 51 activity of a CRISPR-Cas system.

K. pneumoniae clonal group CG147 comprises pandrug- or extensively-resistant

53 isolates and carries multiple and diverse resistance genes and mobile genetic elements, 54 including chromosomal $b l a_{\mathrm{NDM}-5}$. Its emergence is driven by the spread of several 55 phylogenetic clades marked by their own genomic features and specific temporo-spatial 56 dynamics. These findings highlight the need for precision surveillance strategies to limit the 57 spread of particularly concerning CG147 subsets.

59 Data Summary

60 Sequence read files and complete genome assembly of DJ have been deposited in European 61 Nucleotide Archive under the BioProject number PRJEB41234. 


\section{INTRODUCTION}

The increasing number of antimicrobial resistant infections by Klebsiella pneumoniae (Kp), especially by extended-spectrum beta-lactamases (ESBL)- and carbapenemaseproducing $\mathrm{Kp}$, led to the declaration of $\mathrm{Kp}$ as an 'urgent threat' and 'priority pathogen' by public health agencies $(1,2)$. Molecular analyses of $\mathrm{Kp}$ isolates has evidenced that the rapid emergence of multidrug resistant (MDR) Kp is largely driven by the geographic spread of successful clonal groups (CG; e.g., CG15, CG101, CG147, CG258, CG307) (3), some of them carrying epidemic resistance plasmids (4). In order to treat MDR Kp infections, lastresort drugs such as polymyxins (especially colistin) and tigecycline are used $(3,5)$.

Consequently, resistance is also observed to these last resort drugs, especially to colistin, and may culminate in the emergence and spread of pandrug-resistant strains $(5,6)$. Pandrugresistant Kp strains leave few or no therapeutic options and are associated with high mortality rates $(7-11)$.

The 7-gene MLST sequence type (ST) ST147 has been recognized as a globally distributed antimicrobial resistance clone (12), and is closely related to ST273 and ST392, which themselves comprise MDR isolates. Based on genomic classifications, these three STs are grouped into the CG147 clonal group $(13,14)$. The earliest studies of CG147 date from 2008-2010 in Hungary, and correspond to ciprofloxacin-resistant CTX-M-15-producing ST147 isolates, which had been disseminating in the country since $2005(15,16)$. Between 2010 and 2014, CG147 (mainly ST147) was described worldwide in association with several carbapenemases (17). Most reported CG147 isolates are from clinical samples, although some were found in companion animals, river waters, chimpanzees, poultry animals and poultry environment (18-24). The above studies were locally restricted and so far, no study of the global spread and genome dynamics of this clone was performed. 
87 and investigate the genomic evolution and antimicrobial resistance gene dynamics in the 88 global CG147 population. We also analyze the phylogenetic context of virulence-associated 89 genomic features, CRISPR loci and mobile genetic elements in this successful Kp sublineage. 90 
MATERIAL AND METHODS

Isolation and phenotypic and genomic characterization of strain DJ

Isolate DJ was recovered from the urine of a 45-years old female patient diagnosed

with a urinary tract infection (UTI) in Vadodara (Gujarat, India) in October 2016. The isolate

was confirmed to be $\mathrm{Kp}$ by biochemical tests and $16 \mathrm{~S} r R N A$ sequencing. Antibiotic susceptibility tests performed using a semi-automated commercial system (Vitek,

BioMérieux) revealed resistance to all antibiotics tested. Confirmatory antimicrobial susceptibility tests were carried out for colistin and tigecycline using broth dilution; and for

fosfomycin (agar supplemented with $25 \mathrm{mg} / \mathrm{L}$ glucose 6-phosphate sodium salt) using agar

100 dilution method. Disk diffusion method was used for the other antimicrobial classes

101 (penicillins, cephalosporins, carbapenems, monobactams, fluoroquinolones, aminoglycosides, macrolides, tetracyclines, phenicols and inhibitors of the folic acid pathway). Results were interpreted using both the Clinical and Laboratory Standards Institute (25) and the European

Committee on Antimicrobial Susceptibility Testing (2018) (http://www.eucast.org/)

105 guidelines.

106

DNA extraction was performed using XpressDNA Bacteria kit (MagGenome

107 Technologies Pvt Ltd., India). Whole-genome sequencing data were generated using (i) an

108 Illumina NextSeq-500 platform with a 2 x 150 nt paired-end protocol (Nextera XT library,

109 Illumina, San Diego, CA); and (ii) long-read Oxford Nanopore sequencing using MinION

110 device integrated with a FLO-MIN-106 flow cell and libraries prepared using a 1D ligation

111 sequencing kit (SQK-LSK109) following the protocol for 1D genomic DNA (gDNA) long

112 reads without BluePippin (Oxford Nanopore Technologies, New York, NY, USA). De novo

113 assemblies of the reads were obtained using SPAdes v3.12.0 (26) for Illumina data and using

114 Unicycler v0.4.4 (27) for hybrid assembly. Assembled sequences were annotated using 
115 Prokka v1.12 (28). Reads and assembly were deposited at the European Nucleotide Archive database (under the BioProject PRJEB41234).

Global dataset of publicly-available genomic sequences

All publicly available CG147 Kp genomes from NCBI RefSeq repository of genome assemblies (May 2019) were downloaded. From the 245 CG147 genomes available, duplicated $(n=5)$ and poor-quality genomes $(n=6$; genome size and $\mathrm{G}+\mathrm{C}$ content not matching with $\mathrm{Kp}$ and/or more than $>1000$ contigs $)$, and those without attached isolation year $(\mathrm{n}=17)$ were excluded. The final dataset comprised 218 genomes, including strain DJ. Sample information, accession numbers, and biological characteristics of the genomes are given in Table S1.

\section{Phylogenetic analyses}

For phylogenetic analyses, a core-genome alignment based on the concatenation of 4,529 core genes was obtained using Roary v3.12 (29) using a blastP identity cut-off of 90\%

130 and core genes defined as those being present in more than $90 \%$ of the isolates.

131 Recombination events were removed from the core-genome alignment using Gubbins v2.2.0 (30). The final recombination-free alignment comprised 8,450 single-nucleotide variants (SNVs) and was used to construct a maximum-likelihood phylogenetic tree using IQ-TREE

134 v1.6.11 (model GTR+F+ASC+G4). The tree was rooted with a Kp ST258 NJST258_2 135 (accession number: GCF_000597905.1) and a Kp ST37 INF042 (GCF_002752995.1) 136 (Figure S1). To evaluate the strength of the temporal signal of our molecular phylogeny, we first conducted a linear regression analysis of the root-to-tip genetic distances as a function of the 139 sample collection year, using TempEst v1.5.3 (http://tree.bio.ed.ac.uk/software/tempest/) 
140 (Figure S2). The final recombination-free alignment was then subjected to Bayesian

141 phylogenetic analysis using BEAST v2.6.1 (run with a Markov chain Monte Carlo length of

$1421 \times 10^{9}$, sampling every $5 \times 10^{3}$ steps) (31). We used model parameters that had the best fit:

143 GTR substitution model, lognormal relaxed clock and constant population size. Parameters

144 estimates were computed using Tracer v1.7.1, and a maximum clade credibility tree was

145 obtained with TreeAnnotator v2.6.0. and visualized in FigTree v1.4.4.

\section{MLST and genomic analyses of resistance, virulence and other genetic elements}

Multilocus sequence typing (MLST, 7 genes) was performed using the Institut Pasteur

149 Klebsiella MLST (32) database (https://bigsdb.pasteur.fr/klebsiella/). Kleborate (33) and

150 BIGSdb analytical tools (https://bigsdb.pasteur.fr/klebsiella/) (34) were used to define the presence of antimicrobial resistance, heavy metal tolerance, virulence genes and to characterize the capsular gene cluster . Geneious Prime 2019.1.1 software

153 (https://www.geneious.com) was used for further manual curation of antibiotic resistance

154 genes, and ISFinder (https://isfinder.biotoul.fr) was used to look for the insertion sequences

155 in the resistance genes or in their genetic context. Plasmid replicons were detected using 156 PlasmidFinder (https://cge.cbs.dtu.dk/services/PlasmidFinder/) (35), whereas prophages, 157 integrative and conjugative elements (ICEs) and CRISPRs were identified using PHASTER 158 (https://phaster.ca) (36), ICEfinder (https://db-mml.sjtu.edu.cn/ICEfinder/ICEfinder.html) 159 and CRISPRCasFinder (https://crisprcas.i2bc.paris-saclay.fr/CrisprCasFinder/Index) (37),

160 respectively. To depict co-resistance genotypes and plasmid networks, we constructed a 161 correlation matrix for binary variables (1, presence; 0 , absence) using the 'corr.test' function 162 (Pearson method, which for a pair of binary variables compares to the Phi coefficient) from 163 the 'corrplot' R package. Significant correlations were visualized with the 'corrplot' function 164 from the same package. Statistical analyses to check the association of the different 
165 categorical variables within the phylogeny groups were calculated using the $\chi^{2}$ test $(P$ values 166 of $<0.05$ were considered statistically significant). 
RESULTS

\section{Phenotypic and genomic features of pan-drug resistant strain DJ}

Strain DJ was resistant to all tested antimicrobial agents, including last-resort

170 antimicrobials such as carbapenems, colistin, tigecycline and fosfomycin (Table 1), and is

171 therefore pandrug-resistant (6). To define its genetic mechanisms of resistance, a hybrid 172 complete genome assembly was produced (Figure S3a). The 5.7 Mb sequence was 56.9\% $173 \mathrm{G}+\mathrm{C}$-rich and made up of one chromosome and 6 circularized plasmids [123kb 174 IncFII(pKPX1); 57 kb IncR; 5.6 kb ColRNAI; 4.7 kb ColRNAI; 2.0 kb ColpVC; and 1.5 kb 175 ColMG828]. In addition, there were two non-circularized contigs: a 84 kb IncFII plasmid and 176 a $57 \mathrm{~kb}$ contig corresponding to a N15-like phage-plasmid (P-P) coding for a protelomerase 177 (telN) responsible for the maintenance of its linear genome (38). PHASTER identified four 178 other prophages within the chromosome. Phylogenetic analysis of strain DJ showed it belonged to K. pneumoniae sensu stricto (i.e., phylogroup Kp1) and to the ST147-KL64 lineage previously described as endemic in India (12).

The long-read sequencing followed by hybrid assembly of strain DJ allowed the identification of multiple copies of unique antibiotic resistance genes in the different genomic elements depicted (chromosomes and plasmids). Strain DJ harbored four carbapenemase genes, corresponding to two copies of each $b l a_{\mathrm{NDM}-5}$ and $b l a_{\mathrm{OXA}-181}$. Additionally, three copies of bla $_{\mathrm{CTX}-\mathrm{M}-15}$ were detected (Table 1). The above genes were localized as follows (Figure

186 S3, Figure 1): (i) $b l a_{\mathrm{NDM}-5}$ : one copy in the chromosome, and the second copy in the $84 \mathrm{~kb}$

187 IncFII plasmid; (ii) bla $a_{\mathrm{OXA}-181}$ : two copies in the chromosome; and (iii) $b l a_{\mathrm{CTX}-\mathrm{M}-15}$ : two copies in the chromosome and one copy in the $57 \mathrm{~kb}$ IncR plasmid. In addition, a $61 \mathrm{~kb}$ region from the IncFII plasmid, comprising several antimicrobial resistance genes included in

190 a class 1 integron and the replication machinery of the plasmid, was duplicated in the 191 chromosome (FigureS3a,c). 
193 DJ. Most notably, the gene $m g r B$ was disrupted by an IS5 transposase (1057 bp), consistent

194 with the observed colistin resistance phenotype $(\mathrm{MIC}=4 \mu \mathrm{g} / \mathrm{ml})$. This strain also carried $\mathrm{rmtF}$,

$195 r m t B$, strA, strB and aadA2 genes, associated with resistance to aminoglycosides including

196 amikacin (Table 1). Quinolone resistance determining region (QRDR) mutations were

197 observed, leading to GyrA-S83I and ParC-S80I amino acid alterations. Last, there was a

198 premature stop codon caused by a A580T substitution in RamR, a negative regulator of

199 RamA, itself a transcriptional activator of the $a c r A B$ genes. Higher production of AcrAB

200 increases the efflux of tigecycline (39), consistent with the resistance phenotype observed for

201 this agent $(\mathrm{MIC}=16 \mu \mathrm{g} / \mathrm{ml})$.

202 Regarding virulence genes, strain DJ harbored a complete yersiniabactin gene cluster 203 (ybt10) located on an ICEKp4 mobile genetic element (Figure S3a). Type 1 (fimAICDFGH)

204 and type $3(m r k A B C D)$ fimbriae gene clusters were also observed, but none of the $r m p A C D$, 205 aerobactin and salmochelin cluster genes, typically associated with hypervirulence, were 206 present. 
208 Table 1. Antimicrobial susceptibility of Klebsiella pneumoniae strain DJ, and genes

209 potentially conferring resistance.

\begin{tabular}{|c|c|c|c|}
\hline Class and antimicrobial agent & $\begin{array}{l}\text { Diameter } \\
(\mathrm{mm}) \text { or } \mathrm{MIC} \\
(\mu \mathrm{g} / \mathrm{ml})\end{array}$ & Interpretation $^{1}$ & $\begin{array}{l}\text { Associated resistance genes } \\
\text { (copy number) }\end{array}$ \\
\hline Beta-lactams & & & $\begin{array}{l}\text { bla }_{\mathrm{NDM}-5}(2), b l a_{\mathrm{OXA}-181}(2), b l a_{\mathrm{CTX}-\mathrm{M}-15} \\
(3), \text { bla }_{\mathrm{TEM}-1}(2), \text { bla }_{\mathrm{SHV}-11}(1), \text { disrupted } \\
\text { ompK35, mutation in ompK36 }\end{array}$ \\
\hline Ampicillin & 9 & $\mathrm{R}$ & \\
\hline Piperacillin & 13 & $\mathrm{R}$ & \\
\hline Amoxycillin-clavulanic acid & 11 & $\mathrm{R}$ & \\
\hline Ticarcillin-clavulanic acid & 14 & $\mathrm{R}$ & \\
\hline Piperacillin-tazobactam & 14 & $\mathrm{R}$ & \\
\hline Cefuroxime & 11 & $\mathrm{R}$ & \\
\hline Cefotaxime & 12 & $\mathrm{R}$ & \\
\hline Ceftazidime & 15 & $\mathrm{R}$ & \\
\hline Cefepime & 15 & $\mathrm{R}$ & \\
\hline Aztreonam & 14 & $\mathrm{R}$ & \\
\hline Ertapenem & 12 & $\mathrm{R}$ & \\
\hline Imipenem & 14 & $\mathrm{R}$ & \\
\hline Meropenem & 15 & $\mathrm{R}$ & \\
\hline Aminoglycosides & & & $\begin{array}{l}r m t B(1), r m t F(2), \operatorname{aac}\left(6^{\prime}\right)-I b(2), \\
\operatorname{aadA2}(3), \operatorname{str} A B(2)\end{array}$ \\
\hline Amikacin & 10 & $\mathrm{R}$ & \\
\hline Gentamycin & 10 & $\mathrm{R}$ & \\
\hline Tobramycin & 9 & $\mathrm{R}$ & \\
\hline Quinolones and Fluoroquinolones & & & gyrA and parC mutations \\
\hline Nalidixic acid & 11 & $\mathrm{R}$ & \\
\hline Ofloxacin & 11 & $\mathrm{R}$ & \\
\hline Folate pathway inhibitors & & & sull (3), sul2 (1), dfrA12 (3) \\
\hline Trimethoprim/Sulfamethoxazole & 19 & $\mathrm{R}$ & \\
\hline Trimethoprim & 8 & $\mathrm{R}$ & \\
\hline Polymyxins & & & disrupted $m g r B$ \\
\hline Colistin & $\mathrm{MIC}=4$ & $\mathrm{R}$ & \\
\hline Tetracyclines & & & $\operatorname{ram} R$ mutation $^{2}$ \\
\hline Tigecycline & $\mathrm{MIC}=16$ & $\mathrm{R}$ & \\
\hline Phenicols & & & $\operatorname{catA2}(1), \operatorname{catB}(2)$ \\
\hline Chloramphenicol & 12 & $\mathrm{R}$ & \\
\hline \multicolumn{4}{|l|}{ Other } \\
\hline Fosfomycin & $\mathrm{MIC}=128$ & $\mathrm{R}$ & fosA \\
\hline Rifampicin & NI & - & $\operatorname{arr}-2(2)$ \\
\hline Macrolides & $\mathrm{NI}$ & - & $m p h A(1), \operatorname{erm} B(1)$ \\
\hline
\end{tabular}

210 NI, not included in the antimicrobial susceptibility testing panel; R, resistant; ${ }^{1}$ Interpretations were based on CLSI and

211 EUCAST guidelines; ${ }^{2}$ Nucleotide mutation resulting in a premature stop codon. 
We investigated the evolutionary origins of strain DJ within the global diversity of

214 CG147 using 217 publicly available genomes of isolates collected between 2002 and 2018.

215 CG147 genomes were mainly isolated from human samples (90\%, 196/218) and hospital 216 environment $(6 \%, 12 / 218)$, mostly in European (40\%), Southeastern Asia (17\%), Southern

217 Asia (11\%) and Northern America (10\%) countries (Figure 2).

218 CG147 was deeply structured into three main branches (Figure S1), each 219 corresponding to a single MLST sequence type: ST147, ST273 and ST392; the two latter are 220 tonB variants of ST147. The number of genome-wide nucleotide substitutions was associated 221 with isolation dates (root-to-tip regression analysis: $R^{2}=0.1123$, Figure S2), enabling to infer

222 a time-scaled phylogeny (Figure 3). The evolutionary rate within CG147 was estimated at $2231.45 \times 10^{-6}$ substitutions/site/year (95\% HPD, $1.12 \times 10^{-6}-1.78 \times 10^{-6}$ ), corresponding to 6.2 SNPs per genome per year. The last common ancestor of CG147 was estimated around year 1896, with a large uncertainty (95\% highest posterior density (HPD): 1817-1962). The ST273 lineage was the first to diverge, whereas ST147 and ST392 shared a common ancestor, estimated around 1921 (95\% HPD, 1868-1970) (Figure 3).

ST147 was the most represented (79\%, 172/218 genomes) and geographically widespread lineage (Figure 2), and emerged around 1965 (95\% HPD, 1940-1986) (Figure 3). The phylogenetic structure within ST147 revealed five main clades characterized by 231 distinct capsular (KL type) and liposaccharide O antigen loci. Clades KL64-O2 (74\%; 232 128/172) and KL10-O3a (20\%, 34/172) emerged in recent years: 1994 [95\% HPD, 19901998] and 2002 (95\% HPD, 1995-2008), respectively (Figure 3). Whereas KL64-O2 genomes were predominantly from Europe (54\%), KL10-O3a was mainly sampled from Asia (85\%; Figure 2). 
(95\% HPD, 1987-2001), respectively (Figure 3). ST392 (16\% of genomes, 34/218) is

distributed globally and harbors a KL27 capsular gene cluster and a O4 antigen. In contrast,

ST273 $(6 \%, 12 / 218)$ was predominantly found in Asia (64\%) and carries KL74 and O3b gene

240 clusters (Figure 2, Figure 3). Of note, a group of closely related genomes from the

241 Philippines ( $\mathrm{n}=5)$ lacked a capsular gene cluster, with only ugd being detected. This gene had $100 \%$ identity with the $u g d$ gene from the KL74 reference strain, suggesting a recent loss of the capsular gene cluster.

\section{Acquired antimicrobial resistance genes and their evolutionary dynamics within CG147}

All genomes presented QRDR alterations in GyrA and ParC. The topoisomerase ParC

S80I alteration was fully conserved, whereas the GyrA gyrase subunit S83I amino-acid change was observed in all genomes except for clade ST147-KL10, which had S83Y, caused by an ATC to TAC codon change. In addition, this clade had an D87A alteration (Figure 3). genomes ranged from 2 to 23 (mean: 13; Table S1). Regarding beta-lactam resistance, in addition to the conserved chromosomal blasHV-11 gene, a majority of CG147 isolates carried a $b l a_{\text {СтХ-M }}(\mathrm{n}=181,83 \%), 94 \%$ of which was the $b l a_{\text {CTX-M-15 }}$ variant. In addition, $63 \%(\mathrm{n}=137)$ genomes harbored at least one carbapenemase gene, with $14 \%(n=19)$ of these harboring more than one copy of the same carbapenemase gene, and/or two or more carbapenemase genes from different families (Table S1). Noteworthy, carbapenemase genes were significantly more frequent in ST147 (69\%) compared to ST392 (35\%) and ST273 (58\%; $p=0.0005$ ). The two main ST147 clades were similar to this respect (ST147-KL10: 74\%; ST147-KL64: 71\%; $p=0.78$ ). However, their carbapenemase genes were distinct across world regions: there was a predominance of $b l a_{\mathrm{NDM}}$ in Southeastern Asia and Northern America, 
whereas the combination of $b l a_{\mathrm{NDM}}$ and $b l a_{\mathrm{OXA}-48-\mathrm{like}}$ was almost exclusively detected in

262 Southeastern Asia, as observed in strain DJ. In contrast in Europe, bla $a_{\mathrm{OXA}-48-\mathrm{like}}, b l a_{\mathrm{KPC}-2}$ and $b l a_{\mathrm{VIM}-1 / 27}$ were the most frequent carbapenemases (Figure S4; Table S1).

Different $\mathrm{y} b t / \mathrm{ICE} K p$ subtypes (associated with hypervirulence) and OmpK35/K36 mutations (associated with multidrug resistance) were observed within the ST147-KL64 clade (Figure 3; Table S1). First, a group of 29 genomes (denominated subclade 1; mean number of SNPs among them: 57), emerged around 2007, was characterized by the presence of $y b t 16 / \mathrm{ICEKp} 12$ and an altered OmpK35 protein, due to a deletion of 2 nt resulting in a premature stop codon. Within this subclade itself, a subgroup of genomes $(n=19 / 29)$ harbored

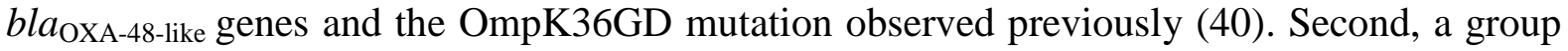
of 22 genomes emerged around 2009 (subclade 2; mean of 60 SNPs) was defined by the presence of $y b t 10 / \mathrm{ICEKp} 4$ and an OmpK35 gene disrupted by ISEcp1-bla CTX-M-15 $_{\text {(Figure 1, }}$

Figure 3, Table S1). As observed in subclade 1, all subclade 2 isolates carrying $b a_{\text {OXA-181 }}$ shared the same OmpK36TD mutation (40). Subclades 1 and 2 also differed in plasmid replicon content: whereas the former were rich in IncR (90\%) and IncHIB/IncFIB(Mar) (41\%), in contrast IncFII (pKPX1) (82\%), IncFII (59\%) and IncR (64\%) were frequent in the latter (Table S1). Differently, the remaining ST147-KL64 genomes $(n=77)$ often carried $\mathrm{IncFIB}_{\mathrm{K}}(65 \%)$, $\mathrm{IncFII}_{\mathrm{K}}(49 \%$; a common pKPN-3-derived plasmid found in Kp harboring pco and sil clusters; $(4,41)$ and IncFIA(HI1) $(38 \%)$.

Strain DJ belonged to subclade 2 and was phylogenetically closely related $(<28$ SNPs) to five other isolates recovered between 2014 and 2015 in different Asian countries

(Figure S3b; Figure3) and described as extremely drug resistant or pandrug-resistant $(11,42,43)$, with identical plasmids being observed among them but no chromosomal integration of $b l a_{\mathrm{NDM}-5}($ Figure S3). 
Whereas the yersiniabactin virulence factor gene cluster was rare amongst genomes of ST392 $(6 \% ; 2 / 34)$ and ST273 $(8 \% ; 1 / 12)$, it was observed in 53\% of ST147 genomes. There were two predominant variants (ybt16/ICEKp 12 and $y b t 10 /$ ICEKp 4 associated with subclades 1 and 2, respectively), and four minority ones (Figure 3; Table S1).

Two isolates with hypervirulence genotypes, defined by the presence of $r m p A$ and/or rmpA2 or aerobactin, were observed: KpvST147L (ST147-KL14, 2016, United Kingdom) and B-8658 (ST147-KL10, 2014, Russia). The plasmid from KpvST147L was fully sequenced previously (GenBank accession number NZ_CM007852) and carries rmpA, rmpA2 and aerobactin; it corresponds to a $343 \mathrm{~kb}$ IncFIB-IncHIB (pNDM-MAR) plasmid (Figure S5) (44). This plasmid was recently described in three ST147 Kp isolates recovered in 2018-2019 in the United Kingdom and in other sublineages (45). Here, we found that Russian strain B-8658 (ST147-KL10, 2014) also acquired the pNDM-MAR plasmid, but it lacked the $r m p A$ gene (Figure S5).

300

Co-occurrence of antimicrobial resistance and heavy metal tolerance genes and plasmids

Antimicrobial resistance genes, mutations and plasmids co-occurred in a structured way (Figure S6; Table S1). For example, Col(BS512), ColKp3, IncFIA (HI1), Cml, EreA/B and GyrA S83Y/D87A co-occurred frequently within the ST147-KL10 clade. Associations of (i) VIM, QnrA1, AadB and IncHI2; (ii) OXA-48-like and IncL(pOXA-48) or (iii) KPC and IncFIB(pQIL) were also observed, consistent with previous descriptions of genetic elements co-carrying these genes (46-48). In addition, genes conferring tolerance to copper and silver were associated with $\mathrm{IncFII}_{\mathrm{K}} / \mathrm{IncFIB}_{\mathrm{K}}$ plasmids ( $p c o$ and sil operons; $p<0.00001$ ), whereas 
association between the tellurite cluster and IncHIB/IncFIB (MAR) was observed $(p<0.0001)$

312 (4). Last, a negative association between IncR and $\mathrm{IncFII}_{\mathrm{K}} / \mathrm{IncFIB}_{\mathrm{K}}$ was observed.

313

314

315

316

317

318

320

321

322

323

324

325

326

327

328

329

330

331

332

333

334

335

\section{CRISPR-Cas systems}

We investigated whether resistance gene dynamics could be influenced by CRISPRCas systems in CG147. There were either one $(83 \% ; 180 / 218)$ or two $(17 \% ; 37 / 218)$ CRISPR-Cas systems amongst CG147 genomes. A conserved subtype IV-E system was found in all genomes, located in the iap - cys $H$ region, with a $\% \mathrm{G}+\mathrm{C}$ of $60.6 \%$, and defined arbitrary as CRISPR1. Direct repeat sequences were highly conserved but the number and sequences of spacers varied across CG147 genomes. CRISPR1 variant v0 (43 spacers) was present in $56 \%$ of the genomes $(122 / 218)$ and was used as reference to define CRISPR1 variants (Figure S7; TableS2). Four of the CRISPR1 v0 spacers (spacers 1, 28, 29 and 42) matched sequences on MDR IncF plasmids disseminated among different Enterobacteriaceae, including Kp. Spacer 1 matched a multicopy intergenic region, whereas spacers 28 and 29 matched a DUF3560-domain containing protein and spacer 42 a hypothetical protein located upstream of SAM-methyltransferase. Although these spacers were highly conserved among CRISPR1 variants, they were also found at high frequency (in $28 \%$ to $53 \%$ of the strains) as protospacers located in plasmid contigs. Noteworthy, in $28 \%$ (62/218) of CG147 genomes these plasmid protospacers were not detected, and the majority of these strains $(65 \%, 40 / 62)$ belonged to subclades 1 and 2 , which were also characterized by the absence of $\operatorname{IncFII}(\mathrm{K})$ and $\operatorname{IncFIB}(\mathrm{KpQIL})$ plasmid replicons. The association of the lack of IncF plasmids with IncF-targeting spacers in CRISPR1, suggests a possible activity of this CRISPR system in subclades 1 and 2. Finally, twelve of the forty-three spacers from CRISPR1 v0 targeted prophages; of these, spacers 6 and 25 were found as protospacers in some of the CG147 genomes (28\% and $18 \%$, respectively). 
Six other CRISPR-Cas systems, distinct from CRISPR1, were observed in 37 genomes. CRISPR2 to CRISPR4, of type IV-A3, were strongly associated with IncHIB and/or IncFIB (pNDM-MAR) plasmids $(p<0.00001)$, as previously described (49). Two variants of CRISPR2 (v1; $\mathrm{n}=12 ; 17$ spacers and $v 3 ; \mathrm{n}=5,25$ spacers) were prevalent (Figure S7; TableS2). CRISPR2 to CRISPR4 systems shared identical direct repeat sequences but showed a high diversity in the number and sequences of spacers. Their tendency to target $\mathrm{IncFII}_{\mathrm{K}} / \mathrm{IncFIB}_{\mathrm{K}}$ plasmids has led to suggest a role in inter-plasmid competition $(49,50)$. However, here the presence of CRISPR2 to CRISPR4 systems was not uniformly associated with an absence of $\mathrm{IncFII}_{\mathrm{K}} / \mathrm{IncFIB}_{\mathrm{K}}$ plasmids.

\section{Prophage elements}

CG147 genomes harbored 0 to 7 prophages (mean of 4 prophages per genome; considering only the intact ones). Some prophages were frequent, including (i) ST147VIM1phi7.1-like (GenBank accession no. NC_049451; Myoviridae family), which was present in 90\% of the genomes (196/218); (ii) Salmonella phage 118970_sal3-like (GenBank accession no. NC_031940; Myoviridae family; 70\% [152/218]); and (iii) Enterobacteria phage mEp237-like (GenBank accession no. NC_019704; Siphoviridae family; 56\% [121/218]) (Figure S7).

The N15-like phage-plasmid (Siphoviridae family) we uncovered in the genomic assembly of strain DJ was present in $37 \%$ of CG147 genomes (81/218). It was strongly associated $(p<0.00001)$ with ST147-KL64 subclades 1 and 2 and present in $92 \%$ of these genomes (Table S1, Figure S7). These two subclades were also enriched in other prophages (mean number of prophages: 5) compared with the remaining CG147 genomes (mean number of prophages=3). 
This study was triggered by the discovery of a pandrug resistance phenotype in strain

363 DJ from India, which prompted us to analyzed its genomic features and understand their 364 dynamics in the context of a large genome dataset of CG147 from multiple world regions. 365 The results revealed its deep phylogenetic structure and a capacity of CG147 members to acquire a wide range of antimicrobial resistance or virulence elements, plasmids and prophages. Of these, Klebsiella capsular locus (KL) switches, which occurred repeatedly, represent prominent phylogenetic markers of CG147 clades. The evolutionary dynamics of these surface structure in CG147 echo those observed in other MDR clonal groups, such as ST258 or ST307 $(13,47,51)$. The multiple clonal expansions of sublineages with distinct KL or O types has clear implications for diagnostic, control or therapeutic strategies such as vaccination and phage therapy. Although its reliance on public genomic sequences may expose this study to a bias towards antibiotic-resistant CG147 isolates, as well as those involved in nosocomial outbreaks, this study provides new insights into the evolutionary history, epidemiology and population dynamics of this important emerging clonal group. The genomic arsenal of antimicrobial resistance features of subclade 2, to which strain DJ belongs, suggest extensively drug and pandrug resistance is not restricted to strain DJ, but rather is a shared characteristic in this particular subclade, already disseminated among Asian countries $(11,42,43,52-54)$. Clonal spread of this subclade between different countries was reported (52). This subclade should be closely monitored and may represent a pioneering situation ushering the worrying prospect of pan-resistance in other CG147 subclades.

In the case of strain DJ, we observed the integration of $b l a_{\mathrm{NDM}-5}$ in the chromosome.

383 Previous studies have reported the one or multiple copies of bla $a_{\mathrm{CTX}-\mathrm{M}-15}$ and $b l a_{\mathrm{OXA}-181}$ integrated into the chromosome of $\mathrm{Kp}$ isolates $(55,56)$, including in the high-risk ST147 385 (52,57). However, the chromosomal integration of bla $a_{\mathrm{NDM}}$ was, to our knowledge, only 
reported once in Kp, in two NDM-1 producing ST14 clinical isolates from Thailand (58). In that case, the chromosomal integration was mediated by IS 5 and the $\operatorname{Tn} 3$ transposase. In strain DJ, the integration of $b l a_{\mathrm{NDM}-5}$ may have been mediated by ISEcp1/IS26 (Figure 1a). The genomic rearrangement due to the replicative transposition of IS26 has previously been shown for IncFII-bla $a_{\mathrm{NDM}-5}$ bearing plasmids (59). The chromosomal incorporation of carbapenemase and ESBL genes is concerning, as it may stabilize these genes by promoting their vertical dissemination (52).

The convergence of MDR and hypervirulence genotypes is being increasingly observed in Kp (3). Here, this worrisome association was detected in two phylogenetically distinct CG147 isolates from 2014 (Russia) and 2016 (United Kingdom), which shared an MDR-Hv IncHIB/FIB plasmid (44). The recent observation of this plasmid in ST101 and ST147 isolates from the United Kingdom (45), with no epidemiological link with the isolate from 2016, suggests its continuous circulation and further risk of horizontal spread.

The ST147-KL64 lineage is globally disseminated. The evolutionary rate we estimated $\left(1.03 \times 10^{-6}\right.$ substitutions/site/year) is very similar to other MDR global sublineages, such as ST258 $\left(1.03 \times 10^{-6}\right.$ substitutions/site/year) and ST307 $\left(1.18 \times 10^{-6}\right.$ 402 substitutions/site/year) (47,51), and slightly slower than the one estimated for ST101 $\left(2.85 \times 10^{-6}\right.$ substitutions/site/year) (60). It is striking that the emergence of ST147-KL64 lineage occurred approximately at the same time as other MDR sublineages ST258 (year 1995), ST307 (1994) and ST101 (1989) (47,51,60). In addition, the presence of GyrA and ParC QRDR alterations is a common characteristic to these MDR high-risk sublineages

407 [CG258 and ST307: GyrA-S83I and ParC-S80I; ST101: GyrA-S83I, GyrA-D87G/N/A and 408 ParC-S80I] $(47,51,60)$. This phenotypic and temporal conjunction points to common drivers and suggests a role of the usage of fluoroquinolones, introduced into clinical practice in the 410 end of the 1980s, in the emergence of MDR Kp sublineages. This is reminiscent of the 
411 scenarios of emergence of Escherichia coli ST131 and methicillin-resistant Staphylococcus aureus ST22 (61-63).

The drivers of genomic diversification of emerging Kp sublineages may include a

414 combination of ecological opportunities to acquire genetic elements, local selective pressure,

415 and molecular mechanisms that enable or restrict genetic flux. Of these, CRISPR-Cas 416 systems may play a role (64-67). In CG258, an association was suggested between the 417 absence of these systems and the ability to acquire IncF plasmids [such as bla $\mathrm{KPC}^{-}$ 418 IncF(pKpQIL-like plasmids)] (68-70). Here we found a conserved type IV-E CRISPR-Cas system (CRISPR1) within CG147, consistent with early reports (9), with four spacers matching IncF plasmid sequences. The distribution of CRISPR1 in the broader Kp species shows a unique association with CG147, with only two exceptions (ST2746 and ST3700; based on $1001 \mathrm{Kp}$ genomes representing unique STs; selected from a dataset of 4222 genomes from NCBI, November 2018; data not shown). Type IV CRISPR-Cas systems primarily target plasmids (50). However, in the majority of CG147 strains, corresponding protospacers were found in plasmid sequences, advocating that the immunity provided by this CRISPR1 system might not be fully functional. In contrast, ST147-KL64 subclades 1 and 2 were largely devoid of these protospacers and did not carry IncFIA, $\operatorname{IncFII}_{K}, \operatorname{IncFIB}_{\mathrm{K}}$ and IncFIB (pQil) plasmids (Figure S7). This observation may suggest a possible activity of the CRIPSR1 system in these recently emerged subclades. Future experimental studies are needed to explore this hypothesis. Noteworthy, it was also among these subclades that the N15-like phage-plasmid was prevalent. Among bacterial phyla, this phage-plasmid family was found almost exclusively in $\mathrm{Kp}$ (38), and we found that $58.9 \%$ of these belonged to CG147. A possible biological role of plasmid-prophages has yet to be established. 
The presence of pandrug-resistant and extremely-drug resistant isolates in CG147,

437 together with the high genetic plasticity and rapid emergence dynamics of this clone, 438 represents a clear threat to public health. CG147 is globally disseminated but shows a strong 439 phylogenetic structure, with different clades being associated with specific genomic features 440 and geographical distributions. These observations underline how different variants of 441 CG147 contribute to the major public health threat posed by Kp, and call for specific 442 surveillance and directed control strategies of this clone and its particularly concerning clades 443 (12).

444 A possible link between the absence of IncF plasmids and the activation of CRISPR1 445 defense system is intriguing. This observation calls for more work on mechanistic drivers of 446 the flux of genetic elements across MDR bacterial lineages. Precise phylogenetic mapping 447 and understanding of the dynamics of antimicrobial resistance features are needed to guide 448 the development of control strategies, especially those that target specific subsets of strains 449 within pathogenic bacterial species, such as CRISPR delivery or toxic conjugation systems $450 \quad(71,72)$. 
452 Ethics approval and consent to participate: To conduct the research, we used bacterial 453 strain DJ, which is not considered a human sample. Accordingly, this research was not 454 considered human research and is out of the scope of the decree $n^{\circ} 2016-1537$ of November 455 16, 2016 implementing law $\mathrm{n}^{\circ}$ 2012-300 of March 5, 2012 on research involving human subjects. Therefore, no ethics approval was needed and no informed consent was required.

Consent for publication: No personal identifying data was used; therefore, no consent was necessary.

Availability of data and materials: All data generated or analysed during this study are 460 included in this published article and its supplementary information files.

461 Competing interests: The authors declare that they have no competing interests.

462 Funding: SD availed a UGC-BSR fellowship for research students and was supported 463 financially by EMBO fellowship sponsored by European Molecular Biology Organization 464 (STF_7993) for a visit in the Brisse lab. CR was supported financially by the MedVetKlebs 465 project, a component of European Joint Programme One Health EJP, which has received 466 funding from the European Union's Horizon 2020 research and innovation programme under 467 Grant Agreement No 773830, and by a Roux-Cantarini grant from Institut Pasteur.

468 Author contributions: S.D. and D.G. coordinated the microbiological cultures of the isolate 469 DJ and its antimicrobial susceptibility testing and biochemical characterization. V.P., C.R. 470 and S.D. performed the genomic sequencing. C.R. designed and coordinated the comparative 471 genomics study, with input from S.D. C.R. wrote the initial version of the manuscript. All 472 authors provided input to the manuscript and reviewed the final version. 
473 Acknowledgments: We thank the Plateforme de Microbiologie Mutualisée (P2M) of Institut 474 Pasteur for Illumina sequencing.

475 Authors license statement: This research was funded, in whole or in part, by Institut Pasteur 476 and by European Union's Horizon 2020 research and innovation programme. For the purpose 477 of open access, the authors have applied a CC-BY public copyright license to any Author 478 Manuscript version arising from this submission. 
481 Figure 1. Genetic context of the different copies of $b l a_{\mathrm{NDM}-5}$ (a), $b l a_{\mathrm{OXA}-181}$ (b) and $b l a_{\mathrm{CTX}-\mathrm{M}-15}$ (c) genes identified in K. pneumoniae strain DJ.

483 Within each panel, the chromosomal and/or plasmid-encoded copies of $b l a_{\mathrm{NDM}-5}, b l a_{\mathrm{OXA}-181}$ 484 and $b l a_{\mathrm{CTX}-\mathrm{M}-15}$ detected in DJ are compared between them. Predicted open reading frames 485 (ORFs) are represented on each line by colored arrows, with arrowheads indicating the direction of transcription: antimicrobial resistance genes (red), mobile genetic elements (MGEs) or gene mobilization-related genes (green); RNA-binding proteins (dark yellow); other functions (light gray). Disrupted genes are outlined with dotted lines. Dark gray blocks connecting the distinct gene regions represent homology levels, as indicated in the gradient key. The nucleotide positions of the represented regions are indicated at the left below the copy descriptors. Figures were created using the Easyfig (https://mjsull.github.io/Easyfig/).

Figure 2. Geographic (main) and temporal (inset) distribution of genomes included in this 494 study. The pie charts represent the frequency of each CG147 clade in each country (see size and color keys). Inset: Bars represent the number of isolates per year for which genome assemblies were available (NCBI RefSeq) as of May 2019, coloured by clade. Red lines represent (solid line) the number of PubMed-indexed records as of March 2020 (identified using the search criteria 'Klebsiella pneumoniae' and 'ST147' or 'ST392' or 'ST273',

500 resulting in a total of 99 distinct entries); and (dotted line) the cumulative number of 501 genomes. The scale on the left $\mathrm{Y}$-axis refers to total number whereas the one on the right 502 refers to cumulative numbers. 
504 Figure 3. Time-scaled phylogeny of 218 CG147 genomes and their epidemiological and 505 molecular characteristics.

506 The phylogeny was obtained using the BEAST tool. The three main branches correspond to 507 the three main sequence types (ST). Tree tips are colored by world region of isolation (see 508 key). Black dots on main nodes indicate $\geq 95 \%$ posterior probability. The grey boxes 509 delineate subclades 1 and 2, as indicated. Capsular (KL) and O-antigen (O) locus types and 510 the yersiniabactin-carrying ICEKp elements are colored according to their variants as shown 511 in the legend. Antimicrobial resistance determinants are indicated by colored rectangles when 512 present. In the antimicrobial resistance determinants columns: ${ }^{1}$ dark pink indicates bla $_{\mathrm{KPC}-2}$ 513 and light pink indicates $b l a_{\mathrm{KPC}-3 ;}{ }^{2}$ dark pink indicates $b l a_{\mathrm{CTX}-\mathrm{M}-15}$ and dark pink indicates other 514 bla $a_{\text {CTX-м variants; }}{ }^{3}$ dark pink indicates $\operatorname{mgrB}$ mutations and dark pink indicates $p m r B$ 515 mutations. AMGs: aminoglycosides; FPIs: folate pathway inhibitors. 
518 Figure S1. Phylogenetic structure of CG147.

519 The tree was obtained by maximum likelihood analysis (IQ-TREE) based on the final 520 recombination-free alignment of concatenated nucleotide sequence alignments of 4,517 core 521 genes. The tree was rooted with a Kp ST258 NJST258_2 (GCF_000597905.1) and a Kp

522 ST37 INF042 (GCF_002752995.1). Branch lengths represent the number of nucleotide substitutions per site (scale, 0.001 substitution per site). The three main branches are colored by sequence type (ST) and branch tips are colored by world region of isolation (see key). ICEK $p$ elements are colored according to their variants (see key). Subclades 1 and 2 are denoted by rectangle outlines.

Figure S2. Temporal signal in genomic sequences.

530 The linear correlation between year of isolation and root-to-tip distance from the maximum

531 likelihood phylogeny for CG147 genomes collection was calculated using TempEst.

532

Figure S3. Replicons of strain DJ.

534 (a) Visual representation of the hybrid Unicycler assembly graph, obtained using Bandage

535 (Wick et al., 2015). Nine replicons were obtained, including the circularized chromosome, 6

536 circularized plasmids, 1 linear phage-plasmid and 1 non-circularized plasmid contig. The size 537 of replicons is shown.

538 (b) Sequence alignments of the chromosomes of strain DJ and the four closest genomes 539 belonging to subclade 2 (<28 SNPs): MS6671 (GenBank Accession Number: 540 GCA_001455995), DA48896 (GCA_003006175.1); CRKP-1215 (GCA_002786755; 
$541=$ CRKP-2297) and SKGH01 (GCA_001644765.1). Strain DJ was used as reference. The

542 outermost circle is an annotation of the reference strain showing the location of antimicrobial

543 drug resistance genes, ytb/ICEKp, KL64 capsular locus and the IncFII chromosomal

544 integration region. The figure was created using BRIG (Alikhan et al., 2011).

545 (c) Sequence alignments of the non-circular IncFII replicon of strain DJ with the closest

546 plasmids of subclade 2 strains: pCRKP-2297_2 (CP024836.1); pCRKP-1215_2

547 (CP024840.1), MS6671_plasmidE (LN824138.1), SKGH01_p3 (CP015503.1), p48896_1

548 (CP024430.1) and the region integrated in the chromosome of strain DJ. The IncFII non-

549 circularized replicon was used as reference. The outermost circle is an annotation of the

550 reference strain showing the location of antimicrobial drug resistance genes in red. In the

551 table the coverage, identity, size and antimicrobial resistance genes of the plasmids that were

552 used for the comparison. In bold the resistance genes also present in DJ replicon.

553 (d) Sequence alignments of the p48896_1 (CP024430.1) plasmid with the plasmids of the

554 closest strains: pCRKP-2297_2 (CP024836.1); pCRKP-1215_2 (CP024840.1),

555 MS6671_plasmidE (LN824138.1), SKGH01_p3 (CP015503.1), DJ-IncFII non-circularized

556 replicon and DJ-IncR plasmid. p48896_1 was used as reference plasmid. The outermost

557 circle is an annotation of the reference strain showing the location of antimicrobial drug

558 resistance genes in red.

559 (e) Sequence alignments of the $123 \mathrm{~kb}$ IncFII(pKPX1) plasmid of strain DJ with the closest

560 plasmids of subclade 2 strains: pCRKP-2297_1 (CP024835.1), pCRKP-1215_1

561 (CP024839.1), MS6671_plasmidB (LN824135.1), SKGH01_p2 (CP015502.1), p48896_2

562 (CP024431.1). The IncFII(pKPX1) plasmid of strain DJ was used as reference. The

563 outermost circle is an annotation of the reference strain showing the location of antimicrobial

564 drug resistance genes in red. In the table the coverage, identity, size and antimicrobial 
resistance genes of the plasmids that were used for the comparison. In bold the resistance genes also present in DJ replicon.

567

Figure S4. Geographic origins of the genomes included in this study and their associated carbapenemase profile.

570 The pie charts represent the frequency of each carbapenemase profile in each country (see key). CPKP-: no carbapenemase.

572

Figure S5. Sequence alignments of MDR-Hv plasmids identified in CG147 genomes.

574 Comparison of MDR-Hv plasmids identified in CG147 genomes (KpvST147L_NDM and B8658) with the reference virulence plasmid pK2044 (AP006726.1) and a similar plasmid recovered in 2019 (pKpvST147B, CP040726.1), which was used as reference. The outermost circle shows annotations of the reference strain.

578

Figure S6. Correlation between genotypes (Pearson method).

580 Blank squares represent correlations without statistical significance $(\mathrm{p}>0.05)$. The plot was created with the corrplot $\mathrm{R}$ package.

582

Figure S7. Time-scaled phylogeny of CG147 genomes and their heavy metal tolerance genes, plasmid replicons, CRIPSR systems, and prophages profile.

585 The phylogeny was obtained using the BEAST tool. The three main branches correspond to the three main sequence types (ST). Tree tips are colored by world region of isolation (see

587 key). Black dots on main nodes indicate $\geq 95 \%$ posterior probability. The grey boxes 588 delineate subclades 1 and 2, as indicated. Capsular (KL) and O-antigen (O) locus types and 589 the yersiniabactin-carrying ICEKp elements are colored according to their variants as shown 
590 in the legend. The presence of heavy metal tolerance genes, plasmid replicons, CRISPR-Cas

591 systems, protospacers and prophages is indicated. In heavy metal tolerance genes columns,

592 lighter yellow indicates an incomplete operon. In prophages lighter orange indicates an

593 questionable prophage. HMTG, heavy metal tolerance genes.

594

595 Table S1. Epidemiological and genomic characteristics of the 218 CG147 K. pneumoniae 596 genomes analysed in this study.

597

598 Table S2. CRISPR/Cas systems and variants identified within CG147 K. pneumoniae 599 genomes.

600 


\section{REFERENCES}

602 1. CDC. Antibiotic Resistance Threats in the United States, 2013. 2013.

603 2. WHO. Antimicrobial resistance: global report on surveillance 2014. 2014.

604 3. Wyres KL, Lam MMC, Holt KE. Population genomics of Klebsiella pneumoniae. 605 Nature Reviews Microbiology. 2020;18:344-59.

606 4. Navon-Venezia S, Kondratyeva K, Carattoli A. Klebsiella pneumoniae: A major

607 worldwide source and shuttle for antibiotic resistance. FEMS Microbiol Rev. 2017;41:25260875.

609 5. Poirel L, Jayol A, Nordmann P. Polymyxins: Antibacterial Activity, Susceptibility

610 Testing, and Resistance Mechanisms Encoded by Plasmids or Chromosomes. Clin Microbiol 611 Rev. 2017;30:557-96.

612 6. Magiorakos A-P, Srinivasan A, Carey RB, Carmeli Y, Falagas ME, Giske CG, et al.

613 Multidrug-resistant, extensively drug-resistant and pandrug-resistant bacteria: an

614 international expert proposal for interim standard definitions for acquired resistance. Clin 615 Microbiol Infect. 2012;18:268-81.

616 7. de Man TJB, Lutgring JD, Lonsway DR, Anderson KF, Kiehlbauch JA, Chen L, et al. 617 Genomic analysis of a pan-resistant isolate of Klebsiella pneumoniae, United States 2016. 618 MBio. 2018;9.

619 8. Lázaro-Perona F, Sotillo A, Troyano-Hernáez P, Gómez-Gil R, de la Vega-Bueno Á, 620 Mingorance J. Genomic path to pandrug resistance in a clinical isolate of Klebsiella 621 pneumoniae. Int J Antimicrob Agents. 2018;52:713-8. 
622 9. Avgoulea K, Pilato V Di, Zarkotou O, Sennati S, Politi L, Cannatelli A, et al. 623 Characterization of Extensively Drug-Resistant or Pandrug-Resistant Sequence Type 147 and 624 101 OXA-48-Producing Klebsiella pneumoniae Causing Bloodstream Infections in Patients 625 in an Intensive Care Unit. Antimicrob Agents Chemother. 2018;62.

626 10. Guducuoglu H, Gursoy NC, Yakupogullari Y, Parlak M, Karasin G, Sunnetcioglu M, 627 et al. Hospital outbreak of a colistin-resistant, NDM-1- A nd OXA-48-producing Klebsiella 628 pneumoniae: High mortality from pandrug resistance. Microb Drug Resist. 2018;24:966-72.

629 11. Zowawi HM, Forde BM, Alfaresi M, Alzarouni A, Farahat Y, Chong TM, et al. 630 Stepwise evolution of pandrug-resistance in Klebsiella pneumoniae. Sci Rep. 2015;5.

631 12. Peirano G, Chen L, Kreiswirth BN, Pitouta JDD. Emerging antimicrobial-resistant 632 high-risk Klebsiella pneumoniae clones ST307 and ST147. Antimicrob Agents Chemother. $6332020 ; 64$.

634 13. Wyres KL, Wick RR, Judd LM, Froumine R, Tokolyi A, Gorrie CL, et al. Distinct 635 evolutionary dynamics of horizontal gene transfer in drug resistant and virulent clones of 636 Klebsiella pneumoniae. PLoS Genet. 2019;15:e1008114.

637 14. Bialek-Davenet S, Criscuolo A, Ailloud F, Passet V, Jones L, Delannoy-Vieillard AS, 638 et al. Genomic definition of hypervirulent and multidrug-resistant Klebsiella pneumoniae 639 clonal groups. Emerg Infect Dis. 2014;20:1812-20.

640 15. Damjanova I, Toth A, Paszti J, Hajbel-Vekony G, Jakab M, Berta J, et al. Expansion 641 and countrywide dissemination of ST11, ST15 and ST147 ciprofloxacin-resistant CTX-M642 15-type beta-lactamase-producing Klebsiella pneumoniae epidemic clones in Hungary in 643 2005--the new "MRSAs"? J Antimicrob Chemother. 2008;62:978-85. 
644 16. Szilágyi E, Füzi M, Damjanova I, Böröcz K, Szonyi K, Tóth A, et al. Investigation of 645 extended-spectrum beta-lactamase-producing Klebsiella pneumoniae outbreaks in Hungary 646 between 2005 and 2008. Acta Microbiol Immunol Hung. 2010;57:43-53.

647 17. Munoz-Price LS, Poirel L, Bonomo RA, Schwaber MJ, Daikos GL, Cormican M, et 648 al. Clinical epidemiology of the global expansion of Klebsiella pneumoniae carbapenemases. 649 Lancet Infect Dis. 2013;13:785-96.

650 18. Zurfluh K, Nüesch-Inderbinen M, Morach M, Zihler Berner A, Hächler H, Stephan R. 651 Extended-spectrum- $\beta$-lactamase-producing Enterobacteriaceae isolated from vegetables 652 imported from the Dominican Republic, India, Thailand, and Vietnam. Björkroth J, editor. 653 Appl Environ Microbiol. 2015;81:3115-20.

654 19. Ovejero CM, Escudero JA, Thomas-Lopez D, Hoefer A, Moyano G, Montero N, et al. 655 Highly Tigecycline-resistant Klebsiella Pneumoniae sequence type 11 (ST11) and ST147 656 isolates from companion animals. Antimicrob Agents Chemother. 2017;61.

657 20. Sato T, Harada K, Usui M, Tsuyuki Y, Shiraishi T, Tamura Y, et al. Tigecycline 658 Susceptibility of Klebsiella pneumoniae Complex and Escherichia coli Isolates from 659 Companion Animals: The Prevalence of Tigecycline-Nonsusceptible $K$. pneumoniae 660 Complex, Including Internationally Expanding Human Pathogenic Lineages. Microb Drug 661 Resist. 2018;24:860-7.

662 21. Zogg AL, Simmen S, Zurfluh K, Stephan R, Schmitt SN, Nüesch-Inderbinen M. High 663 prevalence of extended-spectrum $\beta$-Lactamase producing enterobacteriaceae among clinical 664 isolates from cats and dogs admitted to a veterinary hospital in Switzerland. Front Vet Sci. $665 \quad 2018 ; 5$.

666 22. Zhang R, Li J, Wang Y, Shen J, Shen Z, Wang S. Presence of NDM in non-E. coli 
667 Enterobacteriaceae in the poultry production environment. J Antimicrob Chemother. $668 \quad 2019 ; 74: 2209-13$

669 23. Suzuki Y, Nazareno PJ, Nakano R, Mondoy M, Nakano A, Bugayong MP, et al.

670 Environmental presence and genetic characteristics of carbapenemase-producing 671 Enterobacteriaceae from hospital sewage and river water in the philippines. Appl Environ 672 Microbiol. 2020;86.

673 24. Baron SA, Mediannikov O, Abdallah R, Kuete Yimagou E, Medkour H, Dubourg G, 674 et al. Multidrug-resistant Klebsiella pneumoniae clones from wild chimpanzees and termites 675 in Senegal. Antimicrob Agents Chemother. 2021;

676 25. CLSI. Performance Standards for Antimicrobial Susceptibility Testing. 26th ed. 677 Wayne P, editor. Clinical and Laboratory Standards Institute; 2016. CLSI Supplement M100678 S26.

679 26. Prjibelski A, Antipov D, Meleshko D, Lapidus A, Korobeynikov A. Using SPAdes 680 De Novo Assembler. Curr Protoc Bioinforma. 2020;70:e102.

681 27. Wick RR, Judd LM, Gorrie CL, Holt KE. Unicycler: Resolving bacterial genome 682 assemblies from short and long sequencing reads. PLoS Comput Biol. 2017;13.

683 28. Seemann T. Prokka: rapid prokaryotic genome annotation. Bioinformatics. $684 \quad 2014 ; 30: 2068-9$.

29. Page AJ, Cummins CA, Hunt M, Wong VK, Reuter S, Holden MTG, et al. Roary: 686 Rapid large-scale prokaryote pan genome analysis. Bioinformatics. 2015;31:3691-3.

687 30. Croucher NJ, Page AJ, Connor TR, Delaney AJ, Keane JA, Bentley SD, et al. Rapid 688 phylogenetic analysis of large samples of recombinant bacterial whole genome sequences 
690 31. Bouckaert R, Vaughan TG, Barido-Sottani J, Duchêne S, Fourment M, Gavryushkina

691 A, et al. BEAST 2.5: An advanced software platform for Bayesian evolutionary analysis.

692 PLoS Comput Biol. 2019;15.

693 32. Diancourt L, Passet V, Verhoef J, Grimont PAD, Brisse S. Multilocus Sequence 694 Typing of Klebsiella pneumoniae Nosocomial Isolates. J Clin Microbiol. 2005;43:4178-82.

695 33. Lam MMC, Wick RR, Watts SC, Cerdeira LT, Wyres KL, Holt KE. A genomic 696 surveillance framework and genotyping tool for Klebsiella pneumoniae and its related species 697 complex. Nat Commun. 2021; 12:4188.

698 34. Jolley KA, Bray JE, Maiden MCJ. Open-access bacterial population genomics: 699 BIGSdb software, the PubMLST.org website and their applications. Wellcome Open Res. $700 \quad 2018 ; 3$.

35. Carattoli A, Zankari E, García-Fernández A, Voldby Larsen M, Lund O, Villa L, et al.

702 In silico detection and typing of plasmids using PlasmidFinder and plasmid multilocus 703 sequence typing. Antimicrob Agents Chemother. 2014;58:3895-903.

704 36. Arndt D, Grant JR, Marcu A, Sajed T, Pon A, Liang Y, et al. PHASTER: a better, 705 faster version of the PHAST phage search tool. Nucleic Acids Res. 2016;44:W16-21.

706 37. Couvin D, Bernheim A, Toffano-Nioche C, Touchon M, Michalik J, Néron B, et al. 707 CRISPRCasFinder, an update of CRISRFinder, includes a portable version, enhanced 708 performance and integrates search for Cas proteins. Nucleic Acids Res. 2018;46:W246-51.

709 38. Pfeifer E, Moura De Sousa JA, Touchon M, Rocha EPC. Bacteria have numerous 710 distinctive groups of phage-plasmids with conserved phage and variable plasmid gene 
712 39. Villa L, Feudi C, Fortini D, Garciá-Fernández A, Carattoli A. Genomics of KPC713 producing Klebsiella pneumoniae sequence type 512 clone highlights the role of RamR and 714 ribosomal S10 protein mutations in conferring tigecycline resistance. Antimicrob Agents 715 Chemother. 2014;58:1707-12.

716 40. Fajardo-Lubián A, Ben Zakour NL, Agyekum A, Qi Q, Iredell JR. Host adaptation 717 and convergent evolution increases antibiotic resistance without loss of virulence in a major 718 human pathogen. PLoS Pathog. 2019;15.

719 41. Rodrigues C, Machado E, Ramos H, Peixe L, Novais Â. Expansion of ESBL720 producing Klebsiella pneumoniae in hospitalized patients: a successful story of international 721 clones (ST15, ST147, ST336) and epidemic plasmids (IncR, IncFIIK). Int J Med Microbiol. $722 \quad 2014 ; 304: 1100-8$.

723 42. Nahid F, Zahra R, Sandegren L. A blaOXA-181-harbouring multi-resistant ST147 724 Klebsiella pneumoniae isolate from Pakistan that represent an intermediate stage towards 725 pan-drug resistance. PLoS One. 2017;12.

726 43. Alfaresi M. Whole Genome Sequencing of Klebsiella pneumoniae Strain Unravels a 727 New Model for the Development of Extensive Drug Resistance in Enterobacteriaceae. Open 728 Microbiol J. 2018;12:195-9.

729 44. Turton JF, Payne Z, Coward A, Hopkins KL, Turton JA, Doumith M, et al. Virulence 730 genes in isolates of Klebsiella pneumoniae from the UK during 2016, including among 731 carbapenemase gene-positive hypervirulent K1-st23 and 'non-hypervirulent' types ST147, 732 ST15 and ST383. J Med Microbiol. 2018;67:118-28. 

virulence plasmids in "high-risk" clones of Klebsiella pneumoniae, including those carrying blaNDM-5. Microorganisms. 2019;7.

46. Coelho A, Piedra-Carrasco N, Bartolomé R, Quintero-Zarate JN, Larrosa N, CornejoSánchez T, et al. Role of IncHI2 plasmids harbouring blaVIM-1, blaCTX-M-9, aac(6')-Ib and qnrA genes in the spread of multiresistant Enterobacter cloacae and Klebsiella pneumoniae strains in different units at Hospital Vall d'Hebron, Barcelona, Spain. Int J Antimicrob Agents. 2012;39:514-7.

47. Bowers JR, Kitchel B, Driebe EM, MacCannell DR, Roe C, Lemmer D, et al. Genomic Analysis of the Emergence and Rapid Global Dissemination of the Clonal Group 258 Klebsiella pneumoniae Pandemic. PLoS One. 2015;10:e0133727.

48. van der Bij AK, Peirano G, Goessens WHF, van der Vorm ER, van Westreenen M, Pitout JDD. Clinical and Molecular Characteristics of Extended-Spectrum- beta-Lactamase-

746 Producing Escherichia coli Causing Bacteremia in the Rotterdam Area, Netherlands. 747 Antimicrob Agents Chemother. 2011;55:3576-8.

49. Newire E, Aydin A, Juma S, Enne VI, Roberts AP. Identification of a Type IV-A CRISPR-Cas System Located Exclusively on IncHI1B/IncFIB Plasmids in Enterobacteriaceae. Front Microbiol. 2020;11. al. Type IV CRISPR-Cas systems are highly diverse and involved in competition between 753 plasmids. Nucleic Acids Res. 2020;48:2000-12.

754 51. Wyres KL, Hawkey J, Hetland MAK, Fostervold A, Wick RR, Judd LM, et al. 755 Emergence and rapid global dissemination of CTX-M-15-associated Klebsiella pneumoniae 
strain ST307. J Antimicrob Chemother. 2019;74:577-81.

757 52. Sonnevend Á, Ghazawi A, Hashmey R, Haidermota A, Girgis S, Alfaresi M, et al. 758 Multihospital occurrence of pan-resistant Klebsiella pneumoniae sequence type 147 with an 759 ISEcp1-directed blaOXA-181 insertion in the $\operatorname{mgrB}$ gene in the United Arab Emirates. 760 Antimicrob Agents Chemother. 2017;61.

761 53. Falcone M, Giordano C, Barnini S, Tiseo G, Leonildi A, Malacarne P, et al. 762 Extremely drug-resistant NDM-9-producing ST147 Klebsiella pneumoniae causing infections 763 in Italy, May 2020. Eurosurveillance. 2020;25.

764 54. Simner PJ, Antar AAR, Hao S, Gurtowski J, Tamma PD, Rock C, et al. Antibiotic 765 pressure on the acquisition and loss of antibiotic resistance genes in Klebsiella pneumoniae. $\mathrm{J}$ 766 Antimicrob Chemother. 2018;73:1796-803.

767 55. Pitout JDD, Peirano G, Kock MM, Strydom KA, Matsumura Y. The global 768 ascendency of OXA-48-type carbapenemases. Clin Microbiol Rev. 2019;33:e00102-19.

769 56. Yoon E-J, Gwon B, Liu C, Kim D, Won D, Park SG, et al. Beneficial Chromosomal 770 Integration of the Genes for CTX-M Extended-Spectrum $\beta$-Lactamase in Klebsiella 771 pneumoniae for Stable Propagation . mSystems. 2020;5.

772 57. Rojas LJ, Hujer AM, Rudin SD, Wright MS, Domitrovic TN, Marshall SH, et al. 773 NDM-5 and OXA-181 beta-lactamases, a significant threat continues to spread in the 774 Americas. Antimicrob Agents Chemother. 2017;61.

775 58. Sakamoto N, Akeda Y, Sugawara Y, Takeuchi D, Motooka D, Yamamoto N, et al. 776 Genomic characterization of carbapenemase-producing Klebsiella pneumoniae with 777 chromosomally carried blaNDM-1. Antimicrob Agents Chemother. 2018;62. 
59. Takeuchi D, Akeda Y, Yoshida H, Hagiya H, Yamamoto N, Nishi I, et al. Genomic

779

780

781

782

783

784

785

786

787

788

789

790

791

792

793

794

795

796

797

798

799 reorganization by IS26 in a blaNDM-5-bearing FII plasmid of Klebsiella pneumoniae isolated from a patient in Japan. J Med Microbiol. 2018;67:1221-4.

60. Roe CC, Vazquez AJ, Esposito EP, Zarrilli R, Sahl JW. Diversity, virulence, and antimicrobial resistance in isolates from the newly emerging Klebsiella pneumoniae ST101 Lineage. Front Microbiol. 2019;10.

61. Stoesser N, Sheppard AE, Pankhurst L, De Maio N, Moore CE, Sebra R, et al. Evolutionary History of the Global Emergence of the Escherichia coli Epidemic Clone ST131. MBio. 2016;7:e02162-15.

62. Holden MTG, Hsu LY, Kurt K, Weinert LA, Mather AE, Harris SR, et al. A genomic portrait of the emergence, evolution, and global spread of a methicillin-resistant Staphylococcus aureus pandemic. Genome Res. 2013;23:653-64.

63. Fuzi M, Rodriguez Baño J, Toth A. Global Evolution of Pathogenic Bacteria With Extensive Use of Fluoroquinolone Agents. Front Microbiol. 2020;11.

64. Ostria-Hernández ML, Sánchez-Vallejo CJ, Ibarra JA, Castro-Escarpulli G. Survey of clustered regularly interspaced short palindromic repeats and their associated Cas proteins (CRISPR/Cas) systems in multiple sequenced strains of Klebsiella pneumoniae. BMC Res Notes. 2015;8.

65. Shen J, Lv L, Wang X, Xiu Z, Chen G. Comparative analysis of CRISPR-Cas systems in Klebsiella genomes. J Basic Microbiol. 2017;57:325-36.

66. Li HY, Kao CY, Lin WH, Zheng PX, Yan JJ, Wang MC, et al. Characterization of CRISPR-Cas systems in clinical Klebsiella pneumoniae isolates uncovers its potential 

association with antibiotic susceptibility. Front Microbiol. 2018;9.

801 67. Kamruzzaman M, Iredell JR. CRISPR-Cas System in Antibiotic Resistance Plasmids 802 in Klebsiella pneumoniae. Front Microbiol. 2020;10.

803 68. Tang Y, Fu P, Zhou Y, Xie Y, Jin J, Wang B, et al. Absence of the type I-E CRISPR-

804 Cas system in Klebsiella pneumoniae clonal complex 258 is associated with dissemination of 805 IncF epidemic resistance plasmids in this clonal complex. J Antimicrob Chemother. $806 \quad 2020 ; 75: 890-5$.

807 69. Zhou Y, Tang Y, Fu P, Tian D, Yu L, Huang Y, et al. The type I-E CRISPR-Cas 808 system influences the acquisition of blaKPC-IncF plasmid in Klebsiella pneumoniae. Emerg 809 Microbes Infect. 2020;9:1011-22.

810 70. Mackow NA, Shen J, Adnan M, Khan AS, Fries BC, Diago-Navarro E. CRISPR-Cas 811 influences the acquisition of antibiotic resistance in Klebsiella pneumoniae. PLoS One. $812 \quad 2019 ; 14$.

813 71. López-Igual R, Bernal-Bayard J, Rodríguez-Patón A, Ghigo JM, Mazel D.

814 Engineered toxin-intein antimicrobials can selectively target and kill antibiotic-resistant 815 bacteria in mixed populations. Nat Biotechnol. 2019;37:755-60.

816 72. Bikard D, Euler CW, Jiang W, Nussenzweig PM, Goldberg GW, Duportet X, et al. 817 Exploiting CRISPR-cas nucleases to produce sequence-specific antimicrobials. Nat 818 Biotechnol. 2014;32:1146-50. 